\title{
Distal coronary artery wire perforation: a simple method for infrequent complication
}

\author{
Adrian Włodarczak ${ }^{1}$, Artur Jastrzębski ${ }^{1}$, Magdalena Łanocha ${ }^{2}$ \\ ${ }^{1}$ Copper Health Centre, Lubin, Poland \\ 2Poznan University of Medical Sciences, Poznan, Poland
}

Coronary artery perforations (CAPs) are rare (around $0.3 \%$ ) in patients undergoing routine percutaneous coronary interventions (PCIs) and they are associated with an increased risk of adverse outcomes. Complex lesion procedures, e.g. chronic total occlusion, bifurcation lesion, and rotablation procedures, increase the possibility of CAP occurrence. Treatment of perforations depends on their severity, graded by the Ellis type classification, and the presence of complications, such as tamponade. Early recognition and proper treatment of different perforation types are crucial. Potential therapies include the use of prolonged balloon occlusion, deployment of covered stents, or even cardiac surgery. For distal wire perforation, the treatment option comprises a variety of embolic approaches including mechanical coils, natural clot (thrombus and fat) or synthetic glue injections. Due to uncommon occurrence of CAP, interventional cardiologists infrequently perform such rescue procedures as coil embolisation for distal vessel perforation. Therefore, familiarity with the technique and equipment is essential to ensure patients' safety. We describe a case of distal artery wire perforation successfully treated using coil embolisation. A 68-year-old man was scheduled for $\mathrm{PCl}$ of the left anterior descending and the first diagonal branch (1,1,1 Medina) (Fig. 1A). After difficulties with crossing the side branch we used guidewire with a hydrophilic coating (SION, ASAHI) (Fig. 1B). The bifurcation was treated successfully with two stents (Culotte technique). Final contrast injection revealed distal diagonal wire perforation (Fig. 1C). When it occurred, the patient was asymptomatic, and no signs of pericardial effusion were noticed. Initially, the patient did not receive any invasive treatment. Throughout the next days he remained haemodynamically stable. Bedside echocardiography confirmed no pericardial effusion on repeat imaging. Two days later the patient revealed atypical chest pain without any satisfactory relief after standard analgesic treatment. Control coronary angiography showed a large cavity formation with visible communication with the cardiac vein (Fig. 1D, E). We decided to provide definitive treatment for distal artery perforation by coil embolisation through a microcatheter. We used a regular 6-F guiding catheter and a dedicated microcatheter (Headway 17, Terumo), which was advanced to the distal diagonal branch, allowing delivery of the first coil (MicroPlex10 $1.5 \mathrm{~mm} / 1 \mathrm{~cm}$, Terumo) (Suppl. Fig. 1A — see journal website). Repeat contrast injection revealed ongoing distal bleeding. Implantation of two additional coils (MicroPlex10 $2 \mathrm{~mm} / 2 \mathrm{~cm}$ and MicroPlex10 $2 \mathrm{~mm} / 4 \mathrm{~cm}$ ) helped to achieve complete haemostasis (Suppl. Fig. 1B, C — see journal website). The patient remained haemodynamically stable throughout the procedure. Because of the presence of some minor symptoms, elective angiography was performed the following day, revealing properly placed coils without any signs of residual leakage (Suppl. Fig. 1D - see journal website). After further uneventful recovery, the patient was discharged from hospital in good clinical condition. Nine months later a control angiography was scheduled, which confirmed good results of the procedure. The patient has been stable and uneventful for three and a half years of follow-up. A distal coronary artery perforation caused by the tip of a guidewire usually does not have such a dramatic presentation as main vessel perforation. Initially, it may remain unnoticed. Small vessel perforations usually cause slow bleeding, not leading to tamponade until hours after the procedure. Spontaneous closure usually occurs with drainage and restoration of haemostasis. Even in the case of small perforation, a close monitoring of haemodynamic status is necessary. However, prevention is the most important issue. During the procedure, guidewire position should be continually monitored and polymer-jacketed or stiff guidewires should be exchanged for soft, workhorse guidewires as soon as possible after lesion crossing. Secondly, early detection and familiarity with treatment options are essential for the patient safety. In our case, the patient was initially managed conservatively due to lack of symptoms. However, when symptoms appeared later, even without any signs of tamponade, we decided to intervene by using brain coils. Coil embolisation through a microcatheter is a relatively simple and effective method for a distal coronary artery wire perforation. Knowledge of this technique seems to be necessary for the interventional cardiologists performing complex lesion PCls.

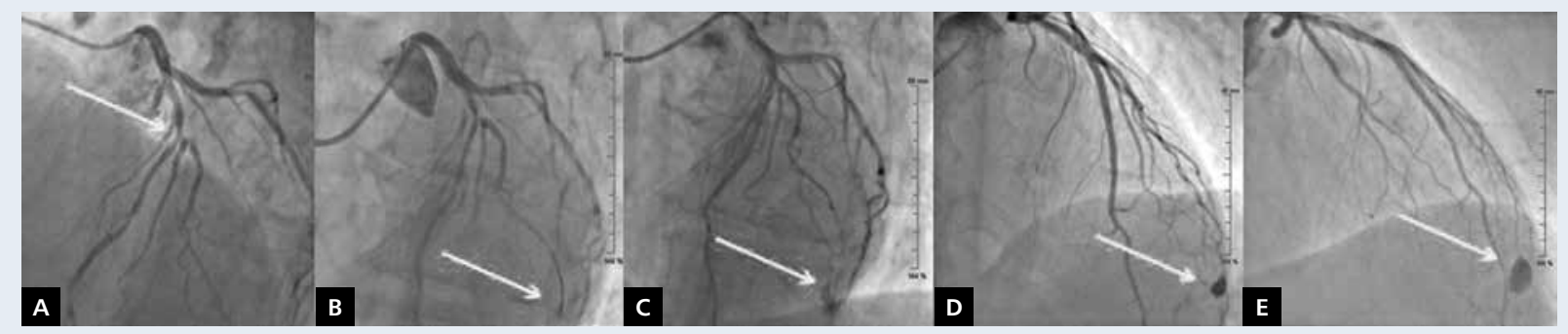

Figure 1. Coronary angiography, bifurcation of the left anterior descending and the first diagonal branch (arrow) (A); looping guidewire at the distal of the side branch (arrow) (B); distal diagonal branch wire perforation (arrow) (C); cavity formation with visible communication with cardiac vein system (arrow) (D); enlargement of cavity two days after percutaneous coronary interventions (arrow) (E)

\section{Address for correspondence:}

Magdalena Łanocha, MD, PhD, $1^{\text {st }}$ Department of Cardiology, Poznan University of Medical Sciences, ul. Długa 1/2, 61-848 Poznań, Poland, tel:+48 61 8549146, fax: +48618549094, e-mail: mlanocha@hotmail.com

Conflict of interest: none declared

Kardiologia Polska Copyright (c) Polish Cardiac Society 2018 